\title{
OCCI And PSO-based Optimization And Management System for Grid Maintenance Scheduling
}

\author{
Liu Qian, Liu Wenying \\ School of Electric and Electronics Engineering, \\ North China Electric Power University, \\ Beijing, China \\ e-mail: xnliuqian@qq.com
}

\author{
Gao Guohua \\ Lanzhou Power School, \\ Lanzhou, China \\ e-mail: erge547@126.com
}

\begin{abstract}
Smart grid is an inevitable choice for the future development of the grid. Smart scheduling is one of the cores to build the strong and smart grid in China. Optimization and Management System for Grid Maintenance Scheduling is an in-depth exploration to achieve dispatching digitalization, intellectualization and visualization. This paper gives a rather detailed description about the functionality and implementation of Optimization and Management System for Grid Maintenance Scheduling. With received maintenance schedules as the basic data, OCCI-based data processing techniques and PSO-based optimization methodology are adopted to achieve the standardization and intellectualization of the grid maintenance scheduling. Moreover, the maintenance schedules generated and related information are managed in a standardized way. The design from this paper together with its programs has been applied in practice, achieving satisfying effect.
\end{abstract}

Keywords- Intelligent Dispatching; Maintenance Scheduling; Optimization Methodology; OCCI; PSO

\section{INTRODUCTION}

As the nerve center of power system, the dispatching centre is of great importance to the secure and economic operation of the grid. OCCI And PSO-based Optimization And Management System for Grid Maintenance Scheduling realizes another deepening of the digitization, intellectualization and visualization of the grid, which also plays a significant role in improving security level and staff efficiency under maintenance conditions.

Traditional maintenance schedules are based on month maintenance schedules received from higher and lower departments. Considering load, operation mode of the grid and maintenance condition of equipments together with other factors, the workers who are in charge of maintenance scheduling make appropriate month and day schedules. On the basis of maintenance schedules received from higher and lower departments, this paper describes the design and realization of Optimization And Management System For Grid Maintenance Scheduling. The intellectualization and standardization of maintenance scheduling for power grid is achieved through data processing, schedules optimizing and information managing with the help of computers.

\section{OptimizATION OF MAINTENANCE SCHEDUles}

\section{A. Initializtion of data}

The initial maintenance schedules sent from power transmitting bureau, power supply bureau, VIP customers, distribution \& cable bureau and EHV bureau are used as initial data of maintenance. For initial data is unlimited in content and written manually, its standardization is poor. As a result, initial data needs processing, which is shown in Fig.1.

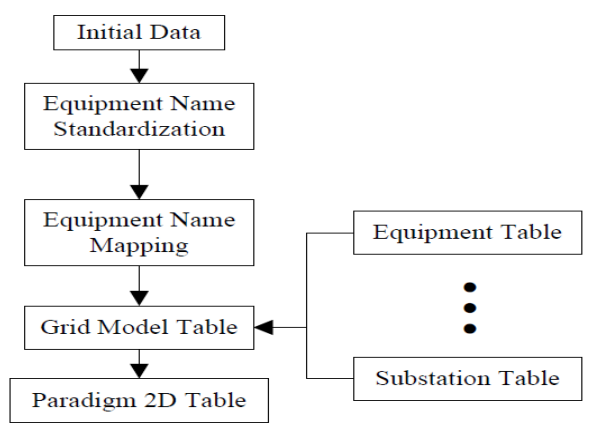

Figure 1. Initial Data Peocessing

Specific steps are as follows.

1) Standardization of device name: According to device name standardization, non-standardized device names are standardized.

2) Code mapping for maintenance device: As the unique identifier, which is the primary key of equipment table, substation table and other tables, the code of device or substation is very important to the management of tables. Code mapping carries on the mapping of device names by searching corresponding codes of device and substation in the grid model library. What is next is to combine the mapped maintained device data, to form a 2NF table with label time (e. g. OLD201103) attached, which will be stored in the Oracle database.

\section{B. Optimization of maintenance scheduling}

As a matter of fact, optimization of maintenance schedules is an optimal planning problem which is based on multi-objective and multi-constraint. Simultaneity constraint, exclusion constraint, season constraint, maintenance resource constraint and safety constraint are taken into 
consideration in the process of monthly maintenance scheduling to improve reliability and economy of the grid.

1) Simultaneity constraint: If the maintenance of several equipments will induce the power interruption of certain load, those equipments should be arranged maintaining at the same time, in order to avoid duplication of power interruption. This constraint involves two principles. On one hand, the downtime of device in power transmitting bureau should be in accordance with that in power supply bureau. On the other hand, the incoming lines in power supply bureau and the outgoing lines in power transmitting bureau should be arranged maintaining at the same time. Field searching method and topology analyzing method are adopted to find devices which meet simultaneity constraint, leaving their downtime adjusted.

2) Exclusion constraint: If the maintenance of some equipment will induce the formation of electrical island, this equipment should be arranged maintaining at the staggered time to prevent avoidable power interruption. The devices which fit exclusion constraint include those which should not be arranged maintaining at the same time according to the predetermined accident plan of district power grid, together with buses, main transformers and tie lines which are spare devices between each other. If there are devices that fit exclusion constraint in maintenance schedules, they should be arranged maintaining at different times.

3) Season constraint: In wet season, all the equipments inside or related to hydropower should not be maintained.

4) Maintenance workload constraint: The workload needed by maintenance should be less than the total amount in the same period. Therefore, there should be less than two lines in power transmitting bureau and no more than two devices in substation to be arranged maintaining in the same period. According to the maintenance workload constraint, the optimization of workload is carried on.

Based on the optimizations above, the PSO (Particle Swarm Optimization) method is adopted, whose principle is shown in (1) and (2), to solve the problem.

$$
\begin{aligned}
& v=v+c_{1} * \operatorname{rand}() *(\text { pbest }- \text { present }) \\
& +c_{2} * \text { rand }() *(\text { gbest }- \text { present }) \\
& \text { present }=\text { present }+v
\end{aligned}
$$

$v$ is the velocity of the particle. present is the current position of the particle. pbest is the individual extreme and gbest is the global extreme. rand() is a random number between 0 and 1. $c_{1}$ and $c_{2}$ are learning factors and usually, $c_{1}=c_{2}=2$.

The optimization goal of this module is to minimize the power loss. With the principle above as constraints, the optimization algorithm is conducted according to PSO, resulting in the adjusted maintenance schedule.

The interface of month maintenance scheduling software is shown in Fig.2.

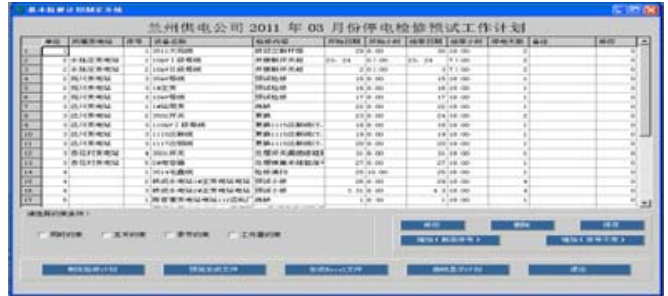

Figure 2. The interface of month maintenance scheduling software

\section{MAINTENANCE INFORMATION MANAGEMENT}

The Oracle database of this system consists of grid model library, maintenance schedules library including basic data, monthly maintenance schedules and daily dispatch prospectuses and maintenance knowledge library including rules of power interruption constraints and topological information of the grid. The data related with the maintenance need to be maintained manually by dispatchers. Consisting of three main parts, which are day maintenance schedules management, month maintenance schedules management and maintenance knowledge library management, the main function of maintenance information management is to manage and maintain the data and information related to the maintenance scheduling. The interface of maintenance information management system is shown in Fig.3.

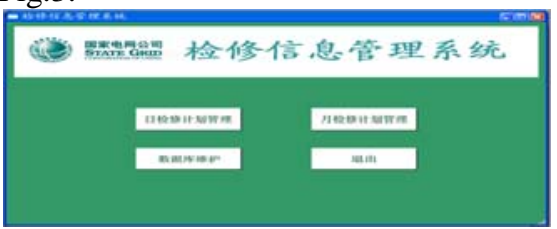

Figure 3. The interface of maintenance information management

Obviously, the main function of day and month maintenance schedules management is to manage and maintain day and month schedules, including querying, modifying and storing, etc. In addition, in the data initializing and maintenance scheduling process, intelligent reasoning is carried on according to the mapping rules and the maintenance rules of the grid, the principles of which also need managing. Maintenance knowledge library management manages and preserves those principles.

The OCCI technology (Oracle $\mathrm{C}++$ Call Interfaces) is a set of application program interface (API) in $\mathrm{C}++$ to access Oracle data. It is designed as a series of well-closed classes and interfaces and first introduced in Oracle9i. It is an upgraded version of OCI (Oracle Call Interfaces) provided by Oracle8i and packs OCI to the object level. It is available to users in the form of a dynamic link library (dll).

In addition, OCCI has many advantages compared with other database access technologies. It provides OCI functions and conducts a comprehensive package of OCI with a consistent interface to call in the form of class, which is simpler than the use of API function. It is easy to be used in $\mathrm{C}++$ and other object-oriented programming. Code 
writing, program understanding and maintenance are more convenient.

Fig.4 illustrates the process of application development based on OCCI

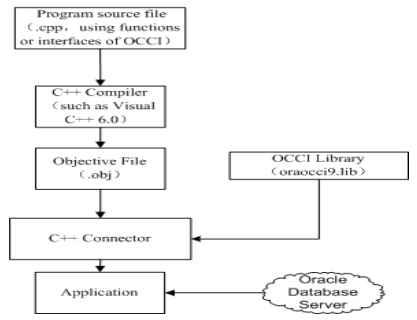

Figure 4. The process of application development based on OCCI

Fig.5 shows the implementation method of data interface based on OCCI technology.

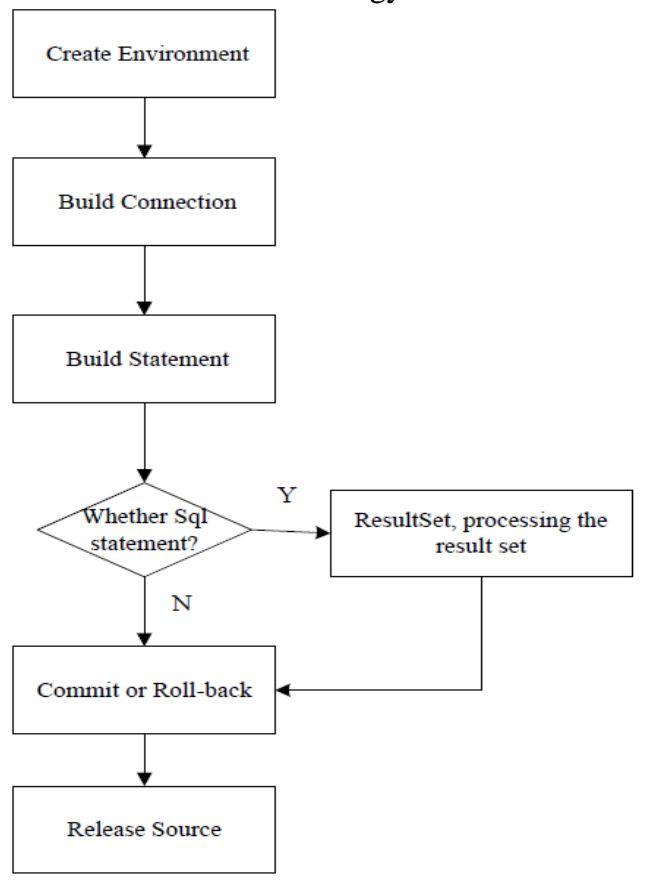

Figure 5. The process to call OCCI interface

(1)Environment: The processing of OCCI exists in this class. It provides the application mode and user-specified memory management function.

(2)Connection: Connection class, created by the Environment object. It is responsible for committing and rolling-back operations.

(3)Statement: This class is responsible for processing SQL statements, generally known as Statement handle, created by the Connection object.

(4)ResultSet: When executing a query statement, the executeQuery method of Statement is used to return a result set object, which holds all records.

Thus, on the basis of a simple, quick, reliable and efficient database interface, programs are written according to the system design. That's how the development of
Information Management System for Maintenance is completed.

\section{IPLEMENTATION OF OptimizATION AND MANAGEMENT SYSTEM FOR GRID MAINTENANCE SCHEDULING}

According to the design above, Optimization And Management System For Grid Maintenance Scheduling has been developed in Visual $\mathrm{C}++6.0$ using $\mathrm{C}++$ language, and has been applied in a regional power grid, with good operation. The implementation process of Optimization And Management System For Grid Maintenance Scheduling is shown in Fig.6.

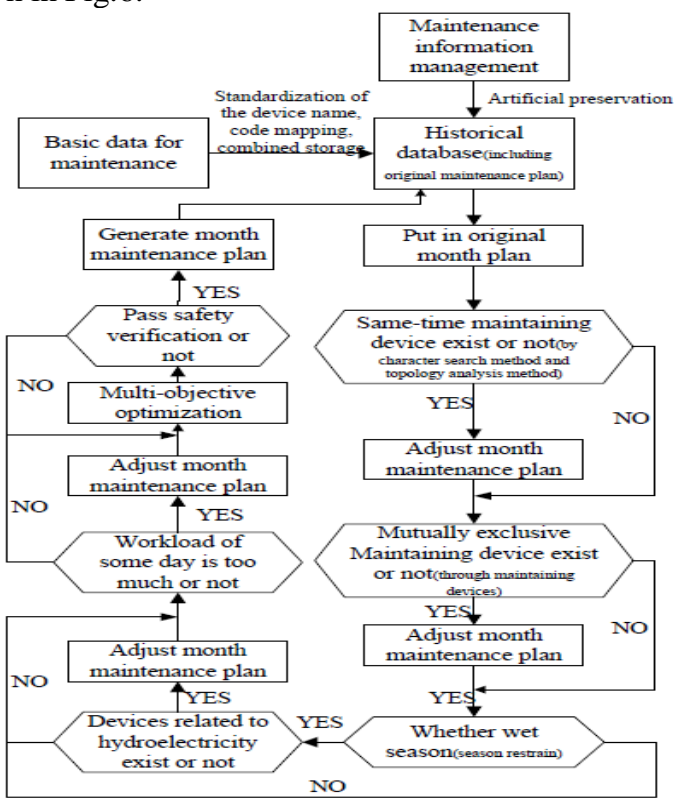

Figure 6. The implementation process of Optimization And Management System For Grid Maintenance Scheduling

\section{Actual Example}

Take the maintenance scheduling in March 2011 at Lanzhou Power Supply Company as an example. The optimized maintenance is scheduled considering multi-object and multi-constraint and then the monthly schedule is distributed to specific branches according to its attached bureau.

Fig.7 shows the initial monthly schedule combining the standardized maintenance data reported by bureaus. Fig.8 shows the optimized month schedule.

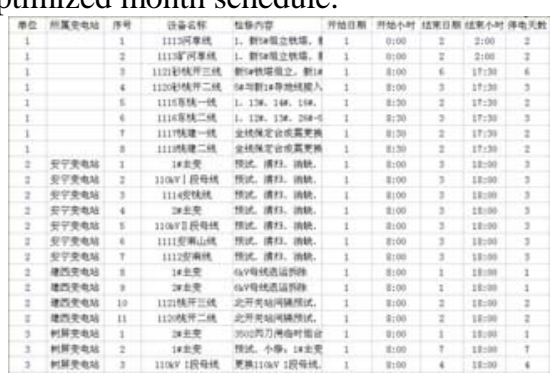

Figure 7. Initial month schedule 


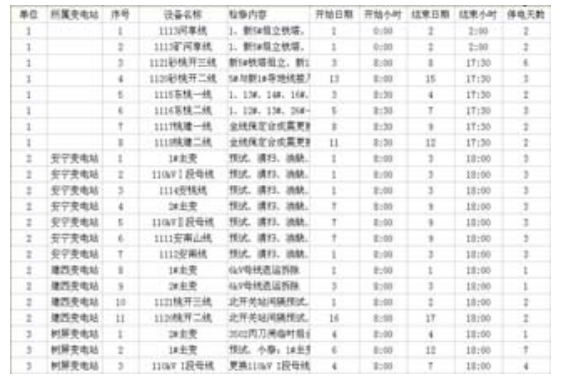

Figure 8. Optimized month schedule

Comparing the results before and after optimization, the maintenance time of devices meeting the constraints has been adjusted, such as Line Shataokai 2 and Line Shataokai 3. Adjusted maintenance schedule meets the security constraints, achieving the desired minimum power loss and ensuring the reliability of the grid.

\section{CONCLUSION}

Optimization and Management System for Grid Maintenance Scheduling adopts the OCCI based data processing method and the PSO based optimization methodology, to achieve the standardized scheduling and optimization of equipment maintenance data. Moreover, this system also provides a standardized management for the generated maintenance schedule and related information through a friendly interactive interface for dispatchers to maintain or adjust those schedules. Thus, the security, economy and reliability to conduct the maintenance schedule of grid are well ensured. This system has already been applied in Lanzhou Power Dispatching Center in Gansu Province, and the actual operation has achieved satisfying results.

\section{REFERENCES}

[1] Wang Zhiming, Researching on the Method of Power Supply System Scheduled Maintenance Basing on Integral Linear Programming. Beijing: North China Electric Power University, 2002.

[2] Zhang Lizi, Huang Xianchao, Shu Jun, and Tao Wenbin, “A design of maintenance scheduling optimization model for distrbution network," Automation of Electric Power Systems. Nanjing, vol. 29, pp. 50-52, 2005.

[3] Zhu Gangyi, Maintenance Scheduling System Considering System Security Constraints. Beijing: North China Electric Power University, 2004

[4] Piao Zailin, Zhao Bin, Liu Na, "Application of genetic algorithm to optimization to the maintenance schedule of rural power network," Transactions of the CSAE. Beijing, vol. 23, pp. 141-145, 2007.

[5] Yang Ning, Wen Fushuan, “Transmission system expansion planning based on chance constrained programming," Automation of Electric Power Systems. Nanjing, 14rd ed., vol. 28, pp. 23-27, 2004.

[6] Dong Lei, Bao Hai, Qi Zheng, one kind of design method for the automatic scheduling system for the maintenance plan in power supply system, vol 18(3), 2001, pp.57-60.

[7] Wang Yingying, Research and Application of Grid Maintenance Scheduling Optimization based on Multi-objective and Multi-constraintLiu Wenying.2010 International Conference on Electrical and Control Engineering.
[8] EI-Sharkh M.Y, EI-Keib A.A., "Maintenance scheduling of generation and transmission systems using fuzzy evolutionary programming,” IEEE Transactions on Power Systems, 2rd ed., vol 18, pp. 862-866, May 2003.

[9] Janjic A.D., Popovic D.S., "Selective maintenance schedule of distribution networks based on risk management approach," IEEE Transaction on Power Systems. Atlanta, 2rd ed., vol. 22, pp. 597-604, May 2007.

[10] Xinju Zhu, Daqi Guo. Optimization of Maintenance Scheduling in Distributed Grid based on Genetic Algoarithm[J]. Electric Switches, 2008,5:25-28. 